\title{
Macular evaluation of the retinal and choroidal vasculature changes in anterior ischemic optic neuropathy-a case control study
}

Hui Wang ${ }^{1+}$, Zhao-yang Meng ${ }^{2 \dagger}$, Song-guo $\mathrm{Li}^{2}$, Jing-jing Wang ${ }^{2}$, Jiao Sun ${ }^{2}$ and Hong-yang $\mathrm{Li}^{2^{*}}$ (D)

\begin{abstract}
Background: This study aimes to characterize the fundus structural changes in patients with nonarteritic anterior ischemic optic neuropathy (NAION) and the correlation between macular vessel density, retinal nerve fibre layer (RNFL) parameters and visual field sensitivity (VFS) in NAION patients.
\end{abstract}

Methods: A retrospective case control study was performed using 37 eyes with NAION, 30 uninvolved contralateral eyes, and 27 eyes of healthy age-matched subjects. Data on the retinas and choroidal vessel densities and VFS were compared among the three groups.

Results: The NAION group exhibited significantly lower RNFL thicknesses, lower ganglion cell complexes (GCC), larger global loss volume (GLV) values and focal loss volume (FLV) values when compared with both uninvolved eyes and healthy eyes ( $p<0.01$ for all comparisons). The superficial vessel density (SVD) valus (whole, parafovea, superior-hemi and inferior-hemi) were significantly lower in NAION eyes, followed by uninvolved eyes and healthy eyes ( $p<0.01$; LSD, $p<0.05$ for all comparisons). The deep vessel density (DVD) values (parafovea, superior-hemi and inferior-hemi) were the lowest by a significant value in NAION eyes, followed by uninvolved eyes and healthy eyes ( $p<0.01$; LSD, $p<0.05$ for all comparisons). However, DVD value measurements (whole and fovea) of healthy and uninvolved eyes were not significantly different. The average threshold deviation (TD) was $-11.02 \pm 3.75 \mathrm{~dB}$ for the overall field region, $-6.01 \pm 2.21 \mathrm{~dB}$ for the affected superior field region and $-9.98 \pm 3.34 \mathrm{~dB}$ for the affected inferior field region in NAION eyes. A statistically significant correlation was found between the RNFL thickness and visual field(VF) loss $(r=-0.788, p<0.001)$.

Conclusion: In addition to peripapillary vascular changes occurring in NAION eyes, macular vessel density is also involved. Furthermore, NAION-uninvolved eyes exhibited abnormalities compared with healthy eyes. This indicates that vascular changes may occur before changes in retinal thickness at the early stages of NAION.

Keywords: Nonarteritic anterior ischemic optic neuropathy, Vessel density, Choroid, Macular

\footnotetext{
*Correspondence: faraway_sweet@163.com

${ }^{+}$Hui Wang and Zhao-yang Meng contributed equally to this work.

${ }^{2}$ Department of Ophthalmology, Beijing Friendship Hospital, the Second

Clinical Medical College of Capital Medical University, No. 95 Yong'an Road,

Xicheng District, Beijing 100050, People's Republic of China

Full list of author information is available at the end of the article
}

(c) The Author(s). 2018 Open Access This article is distributed under the terms of the Creative Commons Attribution 4.0 International License (http://creativecommons.org/licenses/by/4.0/), which permits unrestricted use, distribution, and reproduction in any medium, provided you give appropriate credit to the original author(s) and the source, provide a link to the Creative Commons license, and indicate if changes were made. The Creative Commons Public Domain Dedication waiver (http://creativecommons.org/publicdomain/zero/1.0/) applies to the data made available in this article, unless otherwise stated. 


\section{Background}

Nonarteritic anterior ischemic optic neuropathy (NAION) is the most common acute optic neuropathy and severe visual impairment disease in individuals older than 50 years of age $[1,2]$. The insufficient blood supply of short posterior ciliary arteries (PCA) may lead to anterior optic nerve hypoperfusion, which may cause the occurrence of NAION [3]. The underlying pathophysiology of NAION is unknown; however, it is presumed to be vascular in aetiology and caused by infarction of the retrolaminar portion of the optic nerve head $(\mathrm{ONH})$, which is supplied by the short posterior ciliary arteries [4], although mechanical aetiologies have also been postulated [5].

The potential role of the microvasculature and vessel density in the pathophysiology of NAION has been extensively investigated. Histological studies on optic nerves associated with NAION have shown a complete loss of fibers in one half of the nerve and a peripheral loss of fibers or normal fiber expression in the other half [6]. Multiple studies have demonstrated correlation between visual field threshold and thinning of RNFL following an episode of optic neuritis or NAION [7]. Recently, increasing attention has been paid to the $\mathrm{ONH}$ in NAION diagnostics. Pasol [8] showed in their study that the retinal nerve fiber layer (RNFL) thickness was lower in optic neuropathies than controls. Previous studies have shown that C/D is lower in at risk eyes but becomes larger after NAION episode $[9,10]$. Contreras et al. [11] found in their study that percentages of RNFL loss 3, 6, and 12 months after NAION onset were $38.9,42.3$, and $43.9 \%$, respectively. Moreover, macular involvement was clearly demonstrated in patients with NAION [12]. Nevertheless, little research has been made on the blood flow and structural abnormalities in the macular area in NAION eyes. Although macula is less than $2 \%$ of the retina, it contains $30 \%$ of retinal ganglion cells (RGC).

Recently, the introduction of optical coherence tomography angiography (OCT-A) has allowed for acquisition of various retinal layers, providing a quantitative assessment of the microcirculation of various retinal diseases [13-17]. OCT-A had been used to observe the ONH vessel density in NAIONs. A previous study found that the peripheral vascular density of $\mathrm{ONH}$ in NAIONs decreased significantly [18]. Otherwise, the PCA supplies blood not only to the ONH but also to the choroid.

However, it remains uncertain what role, if any, the macular choroid plays in the pathogenesis of NAION despite some of the shared circulation from the posterior ciliary arteries. It is still unknown, if NAION eyes display abnormal blood flow as compared to the uninvolved fellow eyes and healthy eyes. In order to identify this role, OCTA tends to be more sensitive in detecting subtle changes in NAION eyes to observe whether or not
NAIONs have abnormal blood flow before the onset of clinical symptoms. Our present study assesses the performance of OCT-A macular and retinal vessel density measurements for differentiating among healthy, uninvolved and NAION eyes.

\section{Methods \\ Study design and participants}

This retrospective case control study of healthy, uninvolved, and NAION eyes was performed at Beijing Chaoyang Hospital and Beijing Friendship Hospital, China. A total of 94 eyes were enrolled that completed OCT-A imaging and ONH imaging (AngioVue; Optovue, Inc., CA, USA) with good-quality images. The control group had 33 eyes of healthy age-matched subjects without any ophthalmic pathology. All patients diagnosed with NAION from January 2016 to March 2017 were considered for enrollment in this study. Subjects were divided into three groups: normal control, uninvolved, and NAION. Healthy eyes without NAION, fundus disease, or history of laser therapy or eye surgery were used as the normal controls. The uninvolved group included the 33 contralateral eyes not diagnosed with NAION.

NAION was diagnosed on the basis of some ophthalmologic examination, including detailed history, visual acuity assessment, optic nerve function tests, fundus examination, and visual field defects consistent with NAION. The time lag between the ischemic event and the OCT-A scan was at least 6 months to eliminate the effects of optic disk and RNFL edema observed in the acute phase.

The inclusion criteria were as follows [19]: (1) a history of sudden visual loss, usually discovered in the morning, which was not due to any ocular, systemic, or neurological diseases; (2) optic disk edema at the onset must have been documented in the Ocular Vascular Clinic or by another ophthalmologist; (3) spontaneous resolution of optic disk edema was observed; and (4) the eye had optic disk-related visual field defects.

The exclusion criteria were as follows [20]: (1) obvious retinopathy, including but not limited to high myopia and diabetic retinopathy; evidence of vitreoretinal diseases, such as polypoidal choroidal vasculopathy, ischemic central retinal vein occlusion, or diabetic retinopathy; retinal vascular lesions; medical history of fundus laser and intracranial injection of anti-vascular endothelial growth factor drug; (2) cataract prohibiting adequate retinal evaluation; (3) magnetic resonance imaging suggestive of demyelinating lesions in the brain or spinal cord; (4) evidence of arteritic ischemic optic neuropathy, such as history of scalp tenderness, a significantly elevated sedimentation rate, or elevated C-reactive protein level; (5) a history of inflammatory or infectious diseases (also eliminated by detailed history-taking or relative systemic and laboratory examination); (6) relapse 
of symptoms in the same eye, suggestive of idiopathic demyelinating optic neuritis; and (7) visual field defect larger than one hemifield, spherical equivalent $\geq 3 \mathrm{D}$, and astigmatism $\geq 2$ D. Moreover, participants having an unreliable visual field, poor-quality OCT-A, or ONH scans were also excluded from this study.

The demographic and clinical characteristics including medical history of systemic disease were obtained from participants. Regular ophthalmic examinations and clinical examinations, including measurement of blood pressure (BP) and heart rate, collection of medical and ophthalmologic history, slit-lamp biomicroscopy, visual acuity assessment, intraocular pressure (IOP), dilated examination of fundus, and testing of visual field (VF). OCT-A images were obtained for all the patients by the same operator.

\section{Visual field acquisition}

VFs were assessed with a commercial VF analyser (Humphrey Visual Field Analyzer; Carl Zeiss Meditech, Inc., Dublin, CA) using the Swedish interactive threshold algorithm (SITA) 24-2 Standard protocol. NAION patients with reliable VFs (defined by fixation losses with false-positive and false-negative results amounting to less than $33 \%$ of total results) were included in the study. Because superior and inferior field defects are the most common patterns seen in NAION, we calculated the average threshold deviation (TD) from normal for the superior and inferior regions. The patients were categorized into the following two groups according to locations of VF loss: "inferior field loss (IFL)" and "superior field loss (SFL)". The VFs were averaged based on the decibel $(\mathrm{dB})$ scale, with uniform weighting applied using measurement points.

\section{OCT-A image acquisition and processing}

The AngioVue OCTA system featured in the commercially available Avanti SD-OCT device (RTVue-XR Avanti, Optovue, Fremont, CA) was used to capture the amplitude-decorrelation angiography images. The split-spectrum amplitude-decorrelation angiography (SSADA) algorithm (version 2015.100.0.35) was used to capture the dynamic motion of the red blood cells and to provide a high-resolution 3D visualization of perfused retinal vasculature. Using this software makes it possible to visualize the retinal and choroidal vasculature noninvasively via motion contrast. Vessel density was automatically calculated as the proportion of measured area occupied by flowing blood vessels defined as pixels having decorrelation values acquired by the SSADA algorithm above the threshold level. The motion correction technology software was used to remove saccades and minor loss of fixation [13].
OCT-A imaging was used to measure the superficial vessel density (SVD), deep vessel density (DVD), choroid vessel density (CVD), superficial retinal thickness (SRT) and deep retinal thickness (DRT) in the patients in vivo using the Angio Retina mode $\left(6 \times 6 \mathrm{~mm}^{2}\right)$. The superficial retinal layer extends from the inner limiting membrane with an offset (from the interface reference) of $3 \mu \mathrm{m}$ to the inner plexiform layer with an offset (from the interface reference) of $15 \mu \mathrm{m}$. The deep retinal layer was shown in the slab between 15 and $70 \mu \mathrm{m}$ below the inner plexiform layer. Moreover, the ONH imaging [RNFL thickness and $\mathrm{ONH}$ and ganglion cell complex (GCC) indices measurements] was also analyzed using the RTVue-100 OCT in the optic disk area (ONH protocol, GCC protocol, and 3D Disk) in the tracking mode. The $\mathrm{ONH}$ map protocol was used to obtain the RNFL thickness and $\mathrm{ONH}$ indices. Other indices such as three average GCC parameters (total, superior, and inferior), focal loss volume (FLV), and global loss volume (GLV) were measured in the GCC protocol (Fig. 1, Fig. 2 and Fig. 3).

\section{Statistical analysis}

The Kolmogorov-Smirnov test was used to identify the normality of distribution. Descriptive statistics were calculated as the mean and standard deviation for normally distributed variables and median, first quartile, and third quartile for nonnormally distributed variables. The categorical data were analyzed using the Fisher's exact test. The one-way analysis of variance (ANOVA) test for normal distributions and Kruskal-Wallis tests for nonnormal distributions were used to compare other parameters between groups. The Tamhane's $\mathrm{T}^{2}$ test was performed to adjust for multiple comparisons between groups within each analysis. Spearman rank correlation was used to assess correlations between VF variables and OCTA variables.

The diagnostic accuracy for differentiating between (1) healthy and NAION eyes and (2) healthy and uninvolved eyes were evaluated by calculating the area under the receiver operating characteristic (AUROC) curves. All reported $P$ values were two sided. A $p$ value $<0.05$ was considered statistically significant. Statistical analysis was performed using the SPSS software version 21 (SPSS, Inc., IL, USA). Pairwise comparison of the AUROCs was performed using the method suggested by Pepe al. [21] to evaluate statistical significance between the receiver operating curves.

\section{Results}

This study included 27 healthy eyes, 30 uninvolved eyes, and 37 NAION eyes with good-quality scans. The disease duration of the NAION patients was $9.25 \pm 1.13$ months. No statistically significant differences were found in age, gender, systolic, diastolic, mean BP, and mean ocular perfusion pressure measurements among the groups (Table 1). 


\begin{tabular}{|c|c|c|c|c|}
\hline & \multicolumn{4}{|c|}{ Summary Parameters } \\
\hline \multirow{8}{*}{ 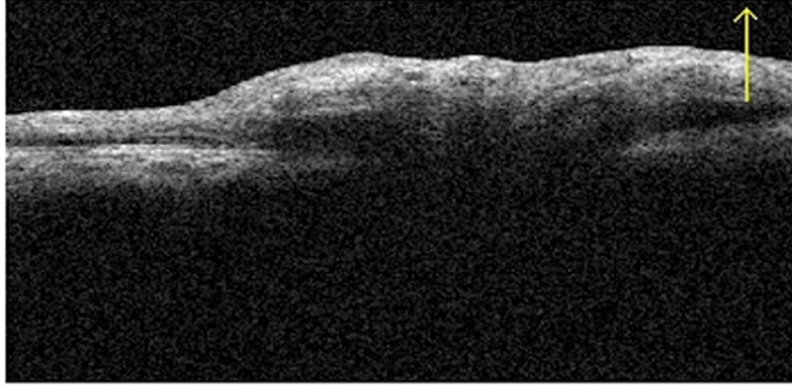 } & RNFL Analysis $(\mu \mathrm{m})$ & OD & OS & Inter Eye (OD-OS) \\
\hline & Avg RNFL Thickness & 75 & 111 & -36 \\
\hline & Avg Superior RNFL & 86 & 109 & -23 \\
\hline & Avg Inferior RNFL & 64 & 113 & -49 \\
\hline & Intra Eye (S-I) & 22 & -4 & N/A \\
\hline & ONH Analysis & OD & OS & Inter Eye (OD-OS) \\
\hline & Cup/Disc Area Ratio & 0.00 & 0.00 & 0.00 \\
\hline & Cup/Disc V. Ratio & 0.00 & 0.00 & 0.00 \\
\hline & Cup/Disc H. Ratio & 0.00 & 0.00 & 0.00 \\
\hline & Rim Area $\left(\mathrm{mm}^{2}\right)$ & 1.66 & 1.70 & -0.04 \\
\hline & Disc Area $\left(\mathrm{mm}^{2}\right)$ & 1.66 & 1.70 & -0.04 \\
\hline 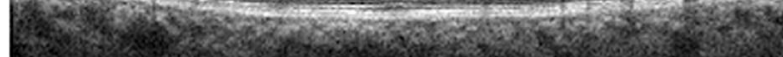 & Cup Volume $\left(\mathrm{mm}^{3}\right)$ & 0.000 & 0.000 & 0.000 \\
\hline & GCC Avg $(\mu \mathrm{m})$ & OD & OS & Inter Eye (OD-OS) \\
\hline & Total & 75.65 & 100.96 & -25.31 \\
\hline & Superior & 76.26 & 99.48 & -23.22 \\
\hline & Inferior & 75.06 & 102.45 & -27.39 \\
\hline $\mathrm{n}>5 \%$ Within Normal & Intra Eye (S-I) & 1.20 & -2.97 & N/A \\
\hline $\mathrm{p}<5 \%$ Borderline & $\mathrm{FLV}(\%)$ & 6.852 & 0.808 & 6.044 \\
\hline $\mathrm{p}<1 \%$ Outside Norma & GLV (\%) & 19.22 & 1.196 & 18.029 \\
\hline $\begin{array}{l}\text { Fig. } 1 \text { RTVue-100 OCT analysed the optic nerve head imaging in the } \\
\text { nerve head (ONH) map protocol was used to obtain RNFL thickness a } \\
\text { parameters (total, superior and inferior), focal loss volume (FLV), and g }\end{array}$ & $\begin{array}{l}\text { c disc area (ONH protocol } \\
\text { ONH indices measuremen } \\
\text { al loss volume (GLV) were }\end{array}$ & $\begin{array}{l}\text { CC pro } \\
\text { Other } \\
\text { asurec }\end{array}$ & ocol and & $\begin{array}{l}\text { 3D Disc) mode. The } \\
\text { ich as three avg. GC } \\
\text { CC protocol }\end{array}$ \\
\hline
\end{tabular}

Ocular and optic nerve characteristics of all the patients IOP was not significantly different among the three groups $(p=0.454)$. The pairwise comparisons of RNFL showed that NAION eyes $(70.86 \pm 10.50 \mu \mathrm{m})$ had significantly lower RNFL thickness values compared with both uninvolved eyes $(98.90 \pm 10.55 \mu \mathrm{m})$ and healthy eyes $(102.11 \pm 7.21 \mu \mathrm{m})$ [least significant difference (LSD), $p$ $<0.001$ for both]. However, the RNFL thickness values was not significantly different between uninvolved and healthy eyes $(p=0.215)$.

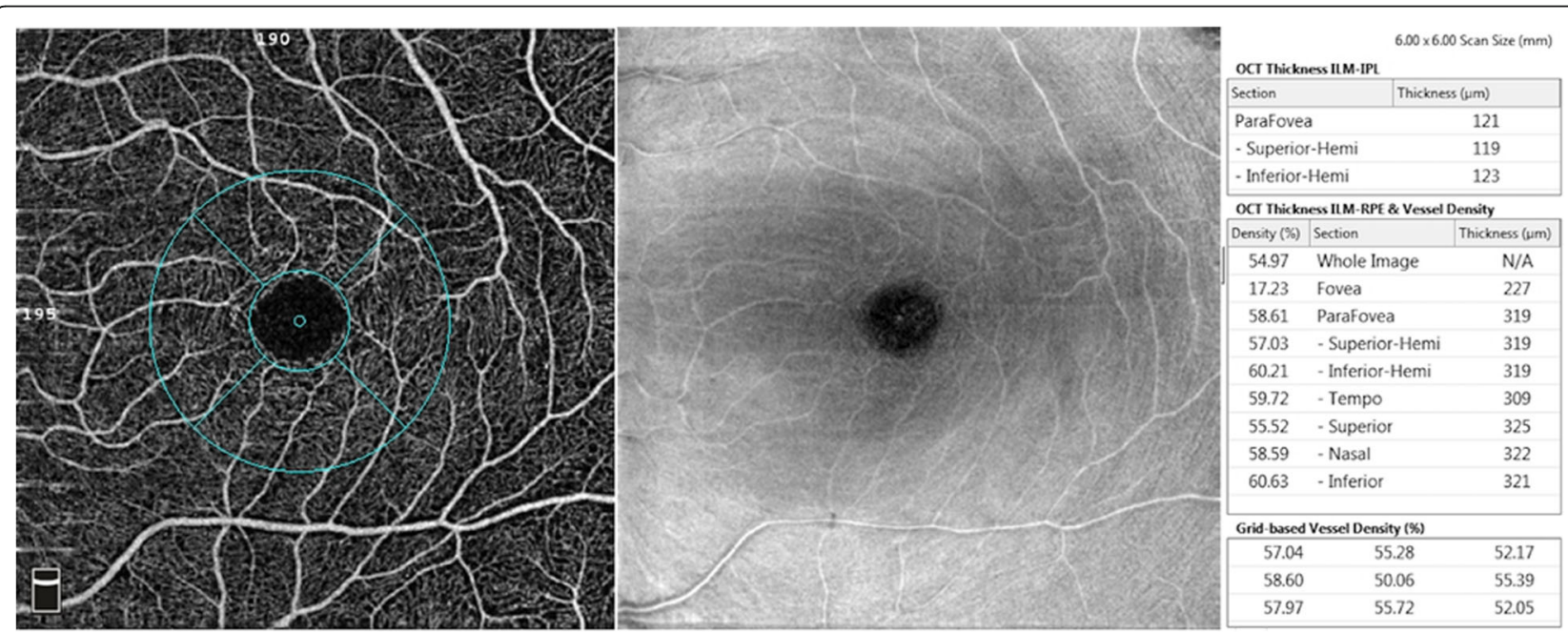

Fig. 2 OCT angiogram of a superficial vascular capillary plexus in a $6 \times 6 \mathrm{~mm}$ area centered on the macula. The en-face image of the superficial plexus was segmented with an inner boundary at $3 \mu \mathrm{m}$ beneath the internal limiting membrane and the outer boundary was set at $15 \mu \mathrm{m}$ beneath the inner plexiform layer 


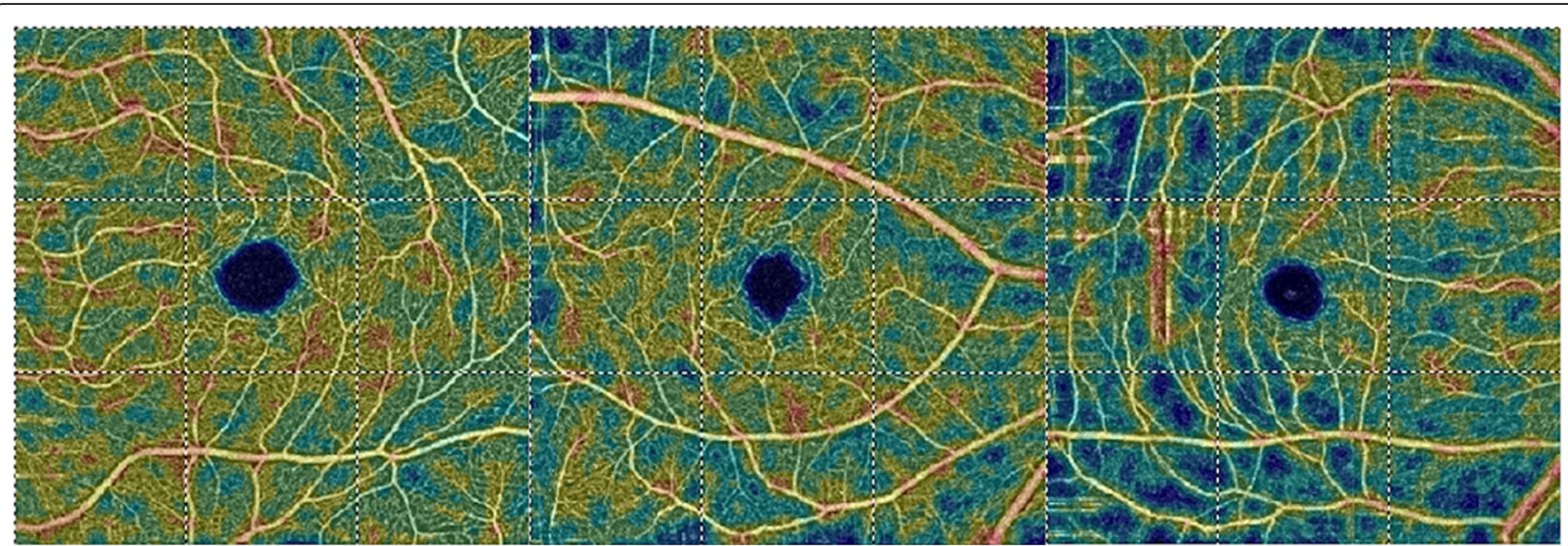

Fig. 3 Retinal superficial vessel density map in healthy, uninvolved, and NAION eyes

Statistically significant differences were found among the three groups. NAION eyes $(72.15 \pm 10.68 \mu \mathrm{m} ; 24.02 \%$ $\pm 8.75 \%$ ) had significantly lower GCC thickness values and larger GLV values compared with both uninvolved eyes $(96.36 \pm 6.82 \mu \mathrm{m} ; 4.56 \% \pm 3.60 \%)$ and healthy eyes $(98.27$ $\pm 7.43 \mu \mathrm{m} ; 3.72 \% \pm 1.70 \%$ ) (LSD, $p<0.001$ for both), but no significant difference was found between uninvolved and healthy eyes $(p=0.408$ and 0.596 , respectively). The FLV values were significantly different among the three groups $(p<0.001)$. NAION eyes $(9.59 \% \pm 3.34 \%)$ had significantly larger FLV values compared with both uninvolved eyes $(2.11 \% \pm 1.65 \%)$ and healthy eyes $(0.86 \% \pm$ $0.40 \%$ ); all three pairwise comparisons were statistically significant (LSD, $p<0.05$ for all comparisons).

The visual field deficits varied, but were typical of NAION. The average TD was $-11.02 \pm 3.75 \mathrm{~dB}$ for overall field region, $-6.01 \pm 2.21 \mathrm{~dB}$ for the affected superior field region and $-9.98 \pm 3.34 \mathrm{~dB}$ for the affected inferior field region in NAION eyes (Table 2).

\section{OCT-A parameters of all the patients}

The ANOVA test of SVD value measurements showed that the vessel density values were significantly different among the three groups $(p<0.001)$. The whole, parafovea, superior-hemi and inferior-hemi values were significantly lower in NAION eyes (44.31, 48.76, 48.72 and $49.05 \%$, respectively), followed by uninvolved eyes (50.55, 54.37, 53.98 and $54.74 \%$, respectively) and healthy eyes $(54.50,56.57,56.37$ and $56.10 \%$, respectively). All three pairwise comparisons were statistically significant (LSD, $p<0.05$ for all comparisons). The deep vessel density (DVD) value measurements (the parafovea, superior-hemi, and inferior-hemi vessel density values) were significantly lower in NAION eyes $(54.36 \% \pm 3.36$,

Table 1 Demographic characteristics of all patients

\begin{tabular}{|c|c|c|c|c|}
\hline Variables & $\begin{array}{l}\text { Healthy } \\
(n=27)\end{array}$ & $\begin{array}{l}\text { Uninvolved eyes } \\
(n=30)\end{array}$ & $\begin{array}{l}\text { NAION eyes } \\
(n=37)\end{array}$ & $P$ Value \\
\hline \multicolumn{5}{|l|}{ Demographic characteristics } \\
\hline Age, year ${ }^{b}$ & $53.70 \pm 9.95$ & $58.17 \pm 13.49$ & $55.05 \pm 7.93$ & $p=0.299$ \\
\hline Gender, male/female & $18 / 9$ & $21 / 9$ & $24 / 13$ & $p=0.905$ \\
\hline Disease duration, months & - & - & $9.25 \pm 1.13$ & \\
\hline \multicolumn{5}{|l|}{ Clinical characteristics } \\
\hline Systolic BP, mm Hg & $128(120,130)$ & $127(120,130)$ & $120(115,130)$ & $p=0.148$ \\
\hline Diastolic BP, mm Hg${ }^{a}$ & $77(75,80)$ & $80(80,80)$ & $80(80,80)$ & $p=0.858$ \\
\hline Heart rate, beats $/ \mathrm{min}^{\mathrm{b}}$ & $79.40 \pm 5.91$ & $79.53 \pm 6.13$ & $77.67 \pm 4.53$ & $p=0.377$ \\
\hline History of diabetes, n (\%) & $5(18.52 \%)$ & $7(23.33 \%)$ & $10(27.03 \%)$ & $p=0.730$ \\
\hline History of hypertension, n (\%) & $6(22.22 \%)$ & $7(23.33 \%)$ & $9(24.32 \%)$ & $p=0.981$ \\
\hline
\end{tabular}

$B P$ Blood pressure, NAION nonarteritic anterior ischemic optic neuropathy

Statistical significance tested by ANOVA for normal distributions and Kruskal-Wallis tests for nonnormal distributions; all comparisons were corrected with the post hoc test. The categorical data were analyzed using the Fisher's exact test of chi-square tests

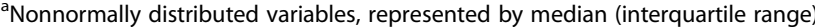

${ }^{b}$ Normally distributed variables, represented by mean ( \pm standard deviation) 
Table 2 Optic nerve characteristics and visual performance of all the patients

\begin{tabular}{|c|c|c|c|c|}
\hline Variables & $\begin{array}{l}\text { Healthy eyes } \\
(n=27)\end{array}$ & $\begin{array}{l}\text { Uninvolved eyes } \\
(n=30)\end{array}$ & $\begin{array}{l}\text { NAION eyes } \\
(n=37)\end{array}$ & $P$ Value \\
\hline \multicolumn{5}{|l|}{ Ocular characteristics } \\
\hline $\mathrm{IOP}, \mathrm{mm} \mathrm{Hg}$ & $13.99 \pm 2.90$ & $14.31 \pm 2.00$ & $14.90 \pm 2.50$ & $p=0.454$ \\
\hline RNFL Thickness ( $\mu \mathrm{m})$ & $102.11 \pm 7.21$ & $98.90 \pm 10.55$ & $70.86 \pm 10.50$ & $p<0.001^{*, \neq, \S}$ \\
\hline \multicolumn{5}{|l|}{ GCC Analysis } \\
\hline GCC Thickness ( $\mu \mathrm{m})$ & $98.27 \pm 7.43$ & $96.36 \pm 6.82$ & $72.15 \pm 10.68$ & $p<0.001^{*, \neq, \S}$ \\
\hline FLV (\%) & $0.86 \pm 0.40$ & $2.11 \pm 1.65$ & $9.59 \pm 3.34$ & $p<0.001^{*, t, \neq, \$}$ \\
\hline GLV (\%) & $3.72 \pm 1.70$ & $4.56 \pm 3.60$ & $24.02 \pm 8.75$ & $p<0.001^{*, \neq, \S}$ \\
\hline \multicolumn{5}{|c|}{ VF parameter (Mean $\pm S D, d B)$} \\
\hline MD & N/A & N/A & $-11.02 \pm 3.75$ & - \\
\hline Superior TD & N/A & N/A & $-6.01 \pm 2.21$ & - \\
\hline Inferior TD & N/A & N/A & $-9.98 \pm 3.34$ & - \\
\hline
\end{tabular}

FLV Focal loss volume, GCC ganglion cell complex, GLV global loss volume, IOP intraocular pressure, NAION nonarteritic anterior ischemic optic neuropathy, RNFL retinal nerve fiber layer, $V F$ visual field, $M D$ mean deviation, $d B$ decibels

* Statistical significance tested by ANOVA for normal distributions and Kruskal-Wallis tests for nonnormal distributions, all comparisons were corrected with post hoc test

+ Significant difference between mean values of healthy eyes and uninvolved eyes

₹ Significant difference between mean values of healthy eyes and NAION eyes

$\S$ Significant difference between mean values of uninvolved eyes and NAION eyes

$54.87 \% \pm 3.97,53.95 \% \pm 3.80 \%$, respectively), followed by uninvolved eyes $(59.09 \% \pm 1.97,59.05 \% \pm 2.23,59.13 \% \pm$ $1.97 \%$, respectively) and healthy eyes $(62.08 \% \pm 1.25$, $61.82 \% \pm 1.94,60.75 \% \pm 2.23 \%$, respectively). All three pairwise comparisons were statistically significant (LSD, $p<0.05$ for all comparisons).

The choroid vessel density (CVD) values were significantly different among the three groups $(p<0.001)$ except for the whole and fovea quarters. The comparisons also showed that NAION eyes had lower whole vessel density compared with healthy eyes $(p=0.001,0.006$, and 0.036 , respectively). The aforementioned vessel density values were significantly lower in NAION eyes than in uninvolved eyes $(p<0.05)$; however, no significant differences were found between uninvolved and healthy controls.

The comparison of superficial retinal thickness (SRT) values showed that NAION eyes $(96.43 \pm 14.40 \mu \mathrm{m}$; $95.43 \pm 13.67 \mu \mathrm{m} ; \quad 96.59 \pm 15.65 \mu \mathrm{m})$ had significantly lower parafovea retinal thickness values (96.43 \pm $14.40 \mu \mathrm{m})$ compared with both uninvolved eyes $(111.47$ $\pm 12.14 \mu \mathrm{m})$ and healthy eyes $(111.89 \pm 11.86 \mu \mathrm{m})(p<$ 0.001); the comparison of deep retinal thickness (DRT) values showed that NAION eyes had significantly lower parafovea retinal thickness values $(183.32 \pm 11.86 \mu \mathrm{m})$ compared with both uninvolved $(191.67 \pm 12.31 \mu \mathrm{m})$ and healthy eyes $(193.04 \pm 9.37 \mu \mathrm{m})(p=0.001)$. However, no significant differences were observed between uninvolved and healthy eyes $(p=0.903$ and 0.650 , respectively). (Table 3).

\section{Spearman rank correlation analyses on structural variables and visual field sensitivity}

There was a statistically significant correlation between the RNFL thickness and VF loss $(r=-0.788, s<0.001)$. However, the SVD; DVD and CVD values were not significantly associated with VF loss (Table 4).

\section{Diagnostic accuracy (AUROC)}

The AUROC curve for differentiating between healthy and NAION eyes was the highest for average RNFL thickness (0.99), followed by whole SVD (0.96), whole DVD (0.89), parafovea SRT (0.81), parafovea DRT (0.73), and whole DVD (0.68). Their diagnostic accuracies in whole vessel density (0.90), parafovea vessel density (0.86), and fovea vessel density (0.82) were lower compared with RNFL thickness $(0.96)(p<0.01$ for all). Pairwise comparisons showed that the AUROC of whole vessel density (0.90) was significantly higher compared with fovea vessel density $(0.82)(p<0.05)$, but similar to parafovea vessel density $(0.86)(p>0.05)$ (Table 5$)$.

The AUROC curve for differentiating uninvolved eyes from healthy eyes was the highest for average whole SVD (0.89), followed by average RNFL thickness (0.68) (Fig. 4).

\section{Discussion}

NAION is the most common and visually threatening optic nerve disorder in the middle-aged and elderly population. The present study demonstrated that the OCT-A vessel density measured in the superficial and deep layers of the retina distinguishes among groups of 
Table 3 Mean vessel density and retinal thickness measured in OCT angiography of all the patients

\begin{tabular}{|c|c|c|c|c|}
\hline OCTA Parameters & $\begin{array}{l}\text { Healthy } \\
(n=27)\end{array}$ & $\begin{array}{l}\text { Uninvolved eyes } \\
(n=30)\end{array}$ & $\begin{array}{l}\text { NAION eyes } \\
(n=37)\end{array}$ & $P$ Value \\
\hline \multicolumn{5}{|l|}{ SVD (\%) } \\
\hline Whole & $54.50 \pm 1.34$ & $50.55 \pm 3.27$ & $44.31 \pm 5.35$ & $p<0.001^{*}, t, \neq, s$ \\
\hline Fovea & $29.57 \pm 1.68$ & $25.40 \pm 5.76$ & $23.28 \pm 5.08$ & $p<0.001^{*}, t, \neq$ \\
\hline Parafovea & $56.57 \pm 1.18$ & $54.37 \pm 3.13$ & $48.76 \pm 5.17$ & $p<0.001^{*,+, \neq, s}$ \\
\hline Superior-Hemi & $56.37 \pm 1.72$ & $53.98 \pm 3.55$ & $48.72 \pm 5.50$ & $p<0.001^{*,+, \neq, s}$ \\
\hline Inferior -Hemi & $56.73 \pm 1.51$ & $54.74 \pm 3.20$ & $49.05 \pm 5.07$ & $p<0.001^{*}, t, \neq, \S$ \\
\hline \multicolumn{5}{|l|}{ DVD (\%) } \\
\hline Whole & $60.35 \pm 1.12$ & $60.08 \pm 1.77$ & $57.12 \pm 2.48$ & $p<0.001^{*}, \neq, \S$ \\
\hline Fovea & $31.49 \pm 2.21$ & $30.36 \pm 3.67$ & $26.73 \pm 2.70$ & $p<0.001^{* \neq, \S}$ \\
\hline Parafovea & $62.08 \pm 1.25$ & $59.09 \pm 1.97$ & $54.36 \pm 3.36$ & $p<0.001^{*, t,+, s}$ \\
\hline Superior-Hemi & $61.82 \pm 1.94$ & $59.05 \pm 2.23$ & $54.87 \pm 3.97$ & $p<0.001^{*}, t, \neq, s$ \\
\hline Inferior -Hemi & $60.75 \pm 2.23$ & $59.13 \pm 1.97$ & $53.95 \pm 3.80$ & $p<0.001^{*}, t, \neq, \$$ \\
\hline \multicolumn{5}{|l|}{ CVD (\%) } \\
\hline Whole & $64.12 \pm 0.95$ & $63.94 \pm 1.49$ & $63.33 \pm 1.27$ & $p=0.035^{*}, \neq$ \\
\hline Fovea & $59.39 \pm 1.94$ & $58.78 \pm 2.90$ & $59.07 \pm 4.09$ & $p=0.775^{*}$ \\
\hline Parafovea & $64.97 \pm 1.34$ & $65.68 \pm 1.50$ & $63.21 \pm 2.26$ & $p<0.001^{*}, \neq, \S$ \\
\hline Superior-Hemi & $66.13 \pm 1.65$ & $65.64 \pm 1.76$ & $62.90 \pm 2.34$ & $p<0.001^{*}, \neq, \S$ \\
\hline Inferior -Hemi & $65.56 \pm 1.49$ & $65.73 \pm 1.59$ & $63.15 \pm 1.80$ & $p<0.001^{*}, \neq, \S$ \\
\hline Parafovea SRT (um) & $111.89 \pm 11.86$ & $111.47 \pm 12.14$ & $96.43 \pm 14.40$ & $p<0.001^{*}, \neq, \S$ \\
\hline Parafovea DRT (um) & $193.04 \pm 9.37$ & $191.67 \pm 12.31$ & $183.32 \pm 11.86$ & $p=0.001^{*}, \neq, \S$ \\
\hline
\end{tabular}

NAION Nonarteritic anterior ischemic optic neuropathy, OCT-A optical coherence tomography angiography, SVD superficial vessel density, DVD deep vessel density, CVD choroid vessel density, SRT superficial retinal thickness, DRT deep retinal thickness

* Statistical significance tested by ANOVA for normal distributions and Kruskal-Wallis tests for nonnormal distributions; all comparisons were corrected with the post hoc test

†'Significant difference between mean values of healthy eyes and uninvolved eyes

${ }^{*}$ Significant difference between mean values of healthy eyes and NAION eyes

${ }^{5}$ Significant difference between mean values of uninvolved eyes and NAION eyes

healthy, uninvolved, and NAION participants. Decreased retinal perfusion was found in NAION eyes, a finding that was correlated with retinal thinning. This study was novel in using OCT-A to demonstrate the retinal perfusion changes in optic atrophy after NAION.

OCT has been shown to be useful in quantifying peripapillary RNFL thickness in NAION [22]. It is also an approximation of the actual RGC axon loss because the

Table 4 Spearman rank correlation analyses on RNFL thickness, vessel density and visual field

\begin{tabular}{lll}
\hline Variables & \multicolumn{2}{l}{ VF loss } \\
\cline { 2 - 3 } \cline { 2 - 2 } & \multicolumn{2}{l}{ Spearman rank correlation } \\
\cline { 2 - 3 } & -0.788 & $p$ \\
\hline RNFL thickness (mm) & -0.355 & $<0.001$ \\
Whole SVD (\%) & -0.417 & 0.372 \\
Whole DVD (\%) & -0.265 & 0.128 \\
Whole CVD (\%) & 0.108
\end{tabular}

RNFL retinal nerve fiber layer, SVD superficial vessel density, DVD deep vessel density, CVD choroid vessel density, VF visual field
RNFL contains glia and blood vessels, which may undergo concomitant changes related to ischemia. RNFL loss in NAION patients has been described in several studies. Rebolleda et al. [23] evaluated the anatomic outcomes after systemic steroid treatment in NAION and found no differences when comparing average RNFL loss with un-treated patients. RNFL thickness was significantly lower in NAION patients compared with the uninvolved eyes [24]. In this study, a significant RNFL loss in eyes were found with NAION $(70.86 \pm 10.50 \mu \mathrm{m})$ compared with both uninvolved eyes $(98.90 \pm 10.55 \mu \mathrm{m})$ and healthy eyes $(102.11 \pm 7.21 \mu \mathrm{m})$, consistent with previous findings. The reason for this change was due to the progressive RNFL thinning as the disease progressed toward optic atrophy described by Savini et al. [22] However, no significant difference was found between uninvolved and healthy eyes.

VF defects are also hallmarks of NAION. Multiple studies have demonstrated correlation between VF threshold and RNFL thinning following episodes of optic neuritis or NAION. Danesh-Meyer et al. [25] and Hood et al. [26] 
Table 5 Diagnostic accuracy (AUROC) for OCT-A vessel density and RNFL thickness measurements in all the patients

\begin{tabular}{lllllll}
\hline $\begin{array}{l}\text { Diagnostic } \\
\text { parameters }\end{array}$ & $\begin{array}{l}\text { Healthy eyes } \\
(95 \% \mathrm{Cl})\end{array}$ & $\begin{array}{l}\text { Uninvolved eyes } \\
(95 \% \mathrm{Cl})\end{array}$ & $\begin{array}{l}\text { NAION eyes } \\
(95 \% \mathrm{Cl})\end{array}$ & $p$ Value & $\begin{array}{l}\text { AUROC (SE) NAION } \\
\text { vs. healthy Eyes }\end{array}$ & $\begin{array}{l}\text { AUROC (SE) Uninvolved } \\
\text { eyes vs. healthy Eyes }\end{array}$ \\
\hline Average RNFL, um & $102.1(99.6-105.1)$ & $98.9(96.0-103.1)$ & $70.9(67.7-74.1)$ & $<0.001^{*,+, \neq}$ & $0.99(0.01)$ & $0.68(0.07)$ \\
Parafovea SRT, um & $111.9(107.7-115.8)$ & $111.5(107.3-115.3)$ & $96.4(92.3-101.0)$ & $<0.001^{*,+, \neq}$ & $0.81(0.05)$ & $0.50(0.08)$ \\
Parafovea DRT, um & $193.0(189.5-197.3)$ & $191.7(187.6-196.1)$ & $183.3(179.7-187.3)$ & $0.001^{*,+, \neq}$ & $0.73(0.06)$ & $0.51(0.08)$ \\
OCT-A whole SVD,\% & $54.5(54.0-55.0)$ & $50.5(49.5-51.8)$ & $44.3(42.5-46.0)$ & $<0.001^{*,+, \neq \ddagger}$ & $0.96(0.03)$ & $0.89(0.05)$ \\
OCT-A whole DVD,\% & $60.3(59.9-60.8)$ & $60.1(59.6-60.6)$ & $57.1(56.1-57.8)$ & $<0.001^{*,+, \neq, \S}$ & $0.89(0.04)$ & $0.53(0.08)$ \\
OCT-A whole CVD,\% & $64.1(63.8-64.5)$ & $63.9(63.4-64.5)$ & $63.3(62.9-63.7)$ & 0.079 & $0.68(0.07)$ & $0.53(0.08)$ \\
\hline
\end{tabular}

AUROC Area under the receiver operating characteristic, Cl confidence interval, CVD choroid vessel density, DRT deep retinal thickness, DVD deep vessel density, NAION Nonarteritic anterior ischemic optic neuropathy, OCT-A optical coherence tomography angiography, SE standard error, SRT superficial retinal thickness, SVD superficial vessel density, RNFL retinal nerve fiber layer

"Statistical significance tested by ANOVA; corrected with the post hoc test

${ }^{\dagger}$ Significant difference between mean values of healthy eyes and uninvolved eyes

₹Significant difference between mean values of uninvolved and NAION eyes

${ }^{\S}$ Significant difference between mean values of healthy and uninvolved eyes

observed a thinner NFL in NAION compared with the controls and correlation between the severity of VF loss and peripapillary NFL loss. Our study confirms these previous observations and shows a significant correlation between thinner RNFLs and VF loss in NAION patients. Kupersmith et al. [7] found in their study that abnormal
RNFL birefringence occurs in sectors corresponding to regional VF loss during acute NAION when OCT-derived RNFL shows thickening. This contradicts the above result, possibly because the patients in this study were all in the acute phase of NAION, OCT showing thickening of the RNFL during acute optic nerve edema.

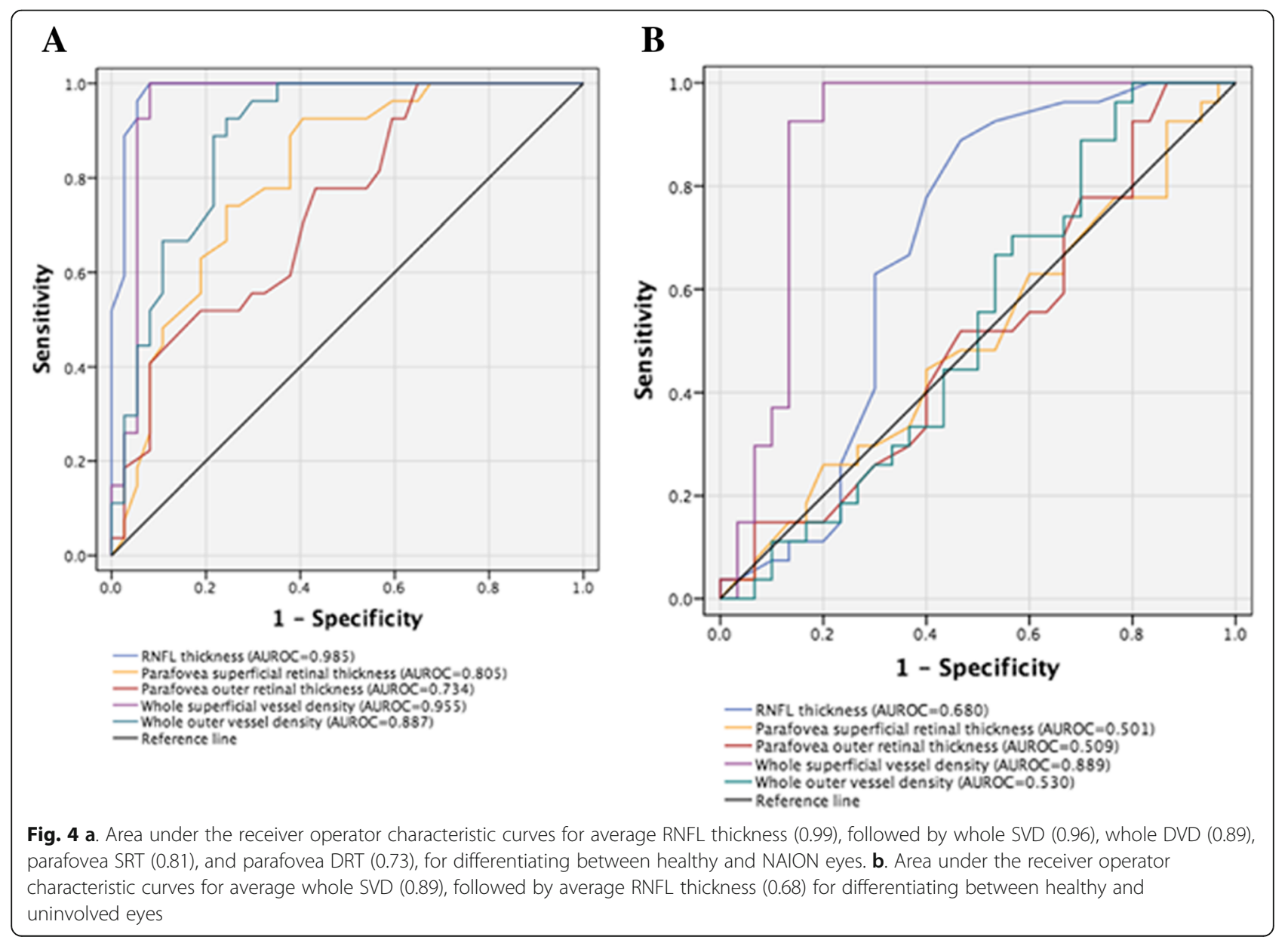


Previous studies about NAION hemodynamic changes focused on the circulatory insufficiency in the $\mathrm{ONH}$; peripapillary retinal perfusion was significantly decreased in optic atrophy after NAION [27]. OCT-A may aid in the understanding of structure-function-perfusion relationships in NAION [27]. Little research was available on macular blood flow in patients with NAION. In this study, the vessel density and retinal thickness both in superficial and deep layers were found to be significantly different among the three groups. OCT-A SVT, DVD, SRT, and DRT were significantly lower in NAION patients compared with fellow uninvolved eyes. Circulatory insufficiency in the $\mathrm{ONH}$ is a widely accepted cause of NAION, which is associated with the dysfunction of short posterior ciliary arteries. The circulatory insufficiency has been found more often in the unaffected eye $[20,28]$. Short posterior ciliary arteries can provide blood supply to the outer layers of retina; the insufficient blood perfusion of short posterior ciliary arteries may result in alterations in the blood supply of the outer layers of retina, which was reflected in this study. Although the mechanism of this outcome is not clear at the moment, the changes in the parameters can provide some evidence in diagnosing NAION. In this study, we also found that the vessel density in the superficial layer decreased in both NAION and uninvolved eyes compared with that of the normal control group of subjects without NAION. Gonul et al. [29] found a thinner subfoveal choroidal thickness (SCT) in both NAION and contralateral eyes in comparison to normal eyes after adjusting ocular and systemic parameters; they hypothesized that a thinner SCT is a potential risk factor for developing NAION. Although the contralateral eye was not diagnosed with NAION, some fundus changes were found. Therefore, we speculate that the abnormal blood supply in the superficial and deep layers had occurred prior to the occurrence of acute posterior ciliary arterial insufficiency and the diagnosis of NAION.

The vessel density both in superficial and deep layers was significantly lower in the uninvolved eyes compared with the healthy groups, a finding worth mentioning. However, the thickness of the superficial and deep retinal layers was not significantly different between the two groups. Compared with the normal subjects, the vessel density was significantly reduced in contralateral uninvolved eyes; however, the corresponding retinal thickness did not change. A reasonable explanation is that the vascular density decreases, reflecting that the blood supply deficiency might occur before the retinal morphologic changes. This vascular density change may be an indicator of early ocular microcirculation disorder before NAION is clinically diagnosed. The development of NAION might occur when the vascular density changes in the uninvolved eyes in NAION patients.
In the present study, the diagnostic accuracy of vessel density measurements was evaluated for differentiating NAION eyes from healthy eyes, as well as uninvolved eyes from healthy subjects. In the NAION and healthy eyes, OCT-A SVD and DVD measures have a similar diagnostic accuracy as RNFL thickness, with AUROCs \pm standard error (SE) of $0.96 \pm 0.03,0.89 \pm 0.04$, and $0.99 \pm$ 0.01 , respectively. The whole SVD was found as a better indicator compared with the RNFL thickness for differentiating uninvolved eyes from healthy subjects, with AUROCs \pm SE of $0.90 \pm 0.05$ and $0.68 \pm 0.07$, respectively. The present study was novel in using OCT-A to differentiate between uninvolved and healthy eyes.

The sample size of this study was relatively small, which may have limited the statistical strength of the analysis. Future studies should be performed with larger cohorts and longer follow-up periods to study changes of retinal choroidal structure in NAION patients. The other limitation was that OCT-A can clearly visualize each layer of retinal capillaries and microvascular status for NAION. However, OCT-A did not show clearly the structural of choroidal vessels, there were measurement errors in choroidal vascular density. Later studies require OCT-A devices with better choroidal imaging quality.

\section{Conclusions}

OCT-A vessel density and retinal thicknesses are lower in NAION patients compared with healthy and uninvolved eyes, and the vessel density has a similar diagnostic accuracy to RNFL thickness for differentiating healthy subjects from NAION patients. It demonstrated that not only peripapillary vessel density of NAION eye had been impaired, but also macular vessel density. Vessel density might occur before retinal thickness changes at an early stage of NAION, making it a sensitive indicator in the early diagnosis of this disease.

\section{Additional file}

Additional file 1: Raw data in this study. (XLSX $69 \mathrm{~kb}$ )

Acknowledgments

Not Applicable.

Funding

This work is supported by "Start Funding of Beijing Friendship Hospital" (Grant Number: yyqdkt2016-8) and was approved by "Beijing Friendship Hospital, the Second Clinical Medical College of Capital Medical University". This funding will not lead to any conflict of interests regarding the publication.

Availability of data and materials

All data generated or analysed during this study are included in this published article [and its Additional file 1].

Authors' contributions

Involved in the design of the study (HY L, HW); conduct of the study (ZY M, SG L, JJ W); collection, management, analysis of the data (HW, ZY M, JS, JJ W); preparation of the manuscript (HW, SG L, ZY M); and critical revision of the manuscript (HY L, HW, SG L). All authors read and approved the final manuscript. 


\section{Ethics approval and consent to participate}

All procedures performed in studies involving human participants were in accordance with the ethical standards of the local ethics committee of Beijing Friendship Hospital and with the 2013 Helsinki declaration. Written informed consent was obtained from the participants in this study.

\section{Consent for publication}

Not applicable.

\section{Competing interests}

The authors declare that they have no competing interests.

\section{Publisher's Note}

Springer Nature remains neutral with regard to jurisdictional claims in published maps and institutional affiliations.

\section{Author details}

'Department of Ophthalmology, Beijing Chaoyang Hospital, the Third Clinical Medical College of Capital Medical University, No. 8 Worker's Stadium East Road, Chaoyang District, Beijing 100043, People's Republic of China. ${ }^{2}$ Department of Ophthalmology, Beijing Friendship Hospital, the Second Clinical Medical College of Capital Medical University, No. 95 Yong'an Road, Xicheng District, Beijing 100050, People's Republic of China.

Received: 10 July 2018 Accepted: 10 December 2018

Published online: 29 December 2018

\section{References}

1. Johnson LN, Arnold AC. Incidence of nonarteritic and arteritic anterior ischemic optic neuropathy. Population-based study in the state of Missour and Los Angeles County, California. J Neuro-Ophthalmol Off J North Am Neuro-Ophthalmol Soc. 1994;14:38-44.

2. Hattenhauer MG, Leavitt JA, Hodge DO, Grill R, Gray DT. Incidence of nonarteritic anterior ischemic optic neuropathy. Am J Ophthalmol. 1997;123: 103-7.

3. Ho SF, Dhar-Munshi S. Nonarteritic anterior ischaemic optic neuropathy. Curr Opin Ophthalmol. 2008;19:461-7.

4. Kerr NM, Chew SSSL, Danesh-Meyer HV. Non-arteritic anterior ischaemic optic neuropathy: a review and update. J Clin Neurosci Off J Neurosurg Soc Australas. 2009:16:994-1000.

5. Parsa CF, Hoyt WF. Nonarteritic anterior ischemic optic neuropathy (NAION): a misnomer. Rearranging pieces of a puzzle to reveal a nonischemic Papillopathy caused by vitreous separation. Ophthalmology. 2015;122:43942.

6. Tesser RA, Niendorf ER, Levin LA. The morphology of an infarct in nonarteritic anterior ischemic optic neuropathy. Ophthalmology. 2003;110: $2031-5$

7. Kupersmith MJ, Anderson S, Durbin M, Kardon R. Scanning laser polarimetry, but not optical coherence tomography predicts permanent visual field loss in acute Nonarteritic anterior ischemic optic neuropathy. Invest Ophthalmol Vis Sci. 2013:54:5514-9.

8. Pasol J. Neuro-ophthalmic disease and optical coherence tomography: glaucoma look-alikes. Curr Opin Ophthalmol. 2011;22:124-32.

9. Saito H, Tomidokoro A, Tomita G, Araie M, Wakakura M. Optic disc and Peripapillary morphology in unilateral Nonarteritic anterior ischemic optic neuropathy and age- and refraction-matched Normals. Ophthalmology. 2008;115:1585-90

10. Contreras I, Rebolleda G, Noval S, Muñoz-Negrete FJ. Optic disc evaluation by optical coherence tomography in Nonarteritic anterior ischemic optic neuropathy. Investig Opthalmology Vis Sci. 2007:48:4087.

11. Contreras I, Noval S, Rebolleda G, Muñoz-Negrete FJ. Follow-up of Nonarteritic anterior ischemic optic neuropathy with optical coherence tomography. Ophthalmology 2007;114:2338-44.e1.

12. Hedges TR, Vuong LN, Gonzalez-Garcia AO, Mendoza-Santiesteban CE, Amaro-Quierza ML. Subretinal fluid from anterior ischemic optic neuropathy demonstrated by optical coherence tomography. Arch Ophthalmol Chic III 1960. 2008:126:812-5.

13. Jia Y, Tan O, Tokayer J, Potsaid B, Wang Y, Liu JJ, et al. Split-spectrum amplitude-decorrelation angiography with optical coherence tomography. Opt Express. 2012;20:4710-25.
14. Rispoli M, Savastano MC, Lumbroso B. Capillary network anomalies in branch retinal vein occlusion on optical coherence tomography angiography. Retina Phila Pa. 2015;35:2332-8.

15. Phasukkijwatana N, Tan ACS, Chen X, Freund KB, Sarraf D. Optical coherence tomography angiography of type 3 neovascularisation in age-related macular degeneration after antiangiogenic therapy. Br J Ophthalmol. 2017; 101:597-602.

16. Agemy SA, Scripsema NK, Shah CM, Chui T, Garcia PM, Lee JG, et al. Retinal vascular perfusion density mapping using optical coherence tomography angiography in normals and diabetic retinopathy patients. Retina Phila Pa. 2015:35:2353-63.

17. Kim AY, Rodger DC, Shahidzadeh A, Chu Z, Koulisis N, Burkemper B, et al. Quantifying retinal microvascular changes in uveitis using spectral-domain optical coherence tomography angiography. Am J Ophthalmol. 2016;171:101-12.

18. Sharma S, Ang M, Najiar RP, Sng C, Cheung CY, Rukmini AV, et al. Optical coherence tomography angiography in acute non-arteritic anterior ischaemic optic neuropathy. Br J Ophthalmol. 2017;101:1045-51.

19. Jonas JB, Hayreh SS, Tao Y, Papastathopoulos KI, Rensch F. Optic nerve head change in non-Arteritic anterior ischemic optic neuropathy and its influence on visual outcome. PLoS One. 2012;7. https://doi.org/10.1371/journal.pone. 0037499.

20. Preechawat P, Bruce BB, Newman NJ, Biousse V. Anterior ischemic optic neuropathy in patients younger than 50 years. Am J Ophthalmol. 2007;144:953-60

21. Pepe $\mathrm{M}$, Longton $\mathrm{G}$, Janes $\mathrm{H}$. Estimation and comparison of receiver operating characteristic curves. Stata J. 2009;9(1).

22. Savini G, Bellusci C, Carbonelli M, Zanini M, Carelli V, Sadun AA, et al. Detection and quantification of retinal nerve fiber layer thickness in optic disc edema using stratus OCT. Arch Ophthalmol Chic III 1960. 2006;124: $1111-7$.

23. Rebolleda G, Pérez-López M, Casas-LLera P, Contreras I, Muñoz-Negrete FJ. Visual and anatomical outcomes of non-arteritic anterior ischemic optic neuropathy with high-dose systemic corticosteroids. Graefes arch Clin Exp Ophthalmol Albrecht Von Graefes Arch Klin Exp Ophthalmol. 2013;251:255-60.

24. Contreras I, Rebolleda G, Noval S, Muñoz-Negrete FJ. Optic disc evaluation by optical coherence tomography in nonarteritic anterior ischemic optic neuropathy. Invest Ophthalmol Vis Sci. 2007;48:4087-92.

25. Daneshmeyer HV, Carroll SC, Ku JY, Hsiang J, Gaskin B, Gamble GG, et al. Correlation of retinal nerve fiber layer measured by scanning laser polarimeter to visual field in ischemic optic neuropathy. Arch Ophthalmol. 2006;124:1720-6.

26. Hood DC, Anderson S, Rouleau J, Wenick AS, Grover LK, Behrens MM, et al. Retinal nerve Fiber structure versus visual field function in patients with ischemic optic neuropathy: a test of a linear model. Ophthalmology. 2008; 115:904-10.

27. Liu C-H, Kao L-Y, Sun M-H, Wu W-C, HS-L C. Retinal vessel density in optical coherence tomography angiography in optic atrophy after Nonarteritic anterior ischemic optic neuropathy. J Ophthalmol. 2017;2017:9632647.

28. Newman NJ, Scherer R, Langenberg P, Kelman S, Feldon S, Kaufman D, et al. The fellow eye in NAION: report from the ischemic optic neuropathy decompression trial follow-up study. Am J Ophthalmol. 2002;134:317-28.

29. Gonul S, Okudan S. Choroidal Thickness in Nonarteritic Anterior Ischemic Optic Neuropathy. Am J Ophthalmol. 2014;158:1342-7.e1.

Ready to submit your research? Choose BMC and benefit from:

- fast, convenient online submission

- thorough peer review by experienced researchers in your field

- rapid publication on acceptance

- support for research data, including large and complex data types

- gold Open Access which fosters wider collaboration and increased citations

- maximum visibility for your research: over $100 \mathrm{M}$ website views per year

At $\mathrm{BMC}$, research is always in progress.

Learn more biomedcentral.com/submissions 